\title{
Determination of Antimicrobial Resistance of Enterobacteriaceae in Patients with Community Acquired Urinary Tract Infections
}

\author{
Abd Elrahman Mustafa Abd Elrahman Osman*, Shingray Osman Hashim, \\ Mohammed Abdall Musa, Omer Mohammed Tahir
}

Medical Laboratory Sciences Division, Port Sudan Ahlia College, Port Sudan, Sudan

Email address:

abdo_rahman96@hotmail.com (A. E. M. A. E. Osman)

*Corresponding author

\section{To cite this article:}

Abd Elrahman Mustafa Abd Elrahman Osman, Shingray Osman Hashim, Mohammed Abdall Musa, Omer Mohammed Tahir. Determination of Antimicrobial Resistance of Enterobacteriaceae in Patients with Community Acquired Urinary Tract Infections. American Journal of Laboratory Medicine. Vol. 2, No. 6, 2017, pp. 179-182. doi: 10.11648/j.ajlm.20170206.20

Received: November 2, 2017; Accepted: November 14, 2017; Published: January 4, 2018

\begin{abstract}
This study was conducted in Port Sudan city, Red Sea state during the period from January to June 2017 to investigate antimicrobial resistance of Enterobacteriaceae isolated from patients suffering from community acquired urinary tract infections. One hundred and fifty urine specimens were collected from patients attended Port Sudan Teaching Hospital. The specimens were cultured on blood agar and Mac Conkey's agar and Cystine Lysine Electrolyte deficient (CLED) agar for primary isolation of pathogens. Identification of the isolates was done by colonial morphology, Gram stain and Routine biochemical tests. Modified Kirby-Bauer disc diffusion method was adopted to determine the resistance rate of Enterobacteriaceae to Imipenem, Ciprofloxacin, Chloramphenicol, Amikacin, Piperacillin, Tetracycline, Ceftazidime and Ticarcillin. Out one hundred and fifty urine specimens examined Enterobacteriaceae was detected in only 49(32.6\%) specimens. The results revealed that the antimicrobial resistance of Enterobacteriaceae was as follows: Imipenem (6.1\%), Ciprofloxacin (32.6\%), Chloramphenicol (48.9\%), Amikacin (61.2\%), Piperacillin (79.5\%), Tetracycline (83.6\%), Ceftazidime $(89.7 \%)$ and Ticarcillin $(91.8 \%)$. Females were more affected than males $(60 \%)$ and young adults were more affected than other age groups. Imipenem represented the least sensitive antimicrobial agent $(6.1 \%)$, while Ticarcillin showed the highest resistance rate.
\end{abstract}

Keywords: Enterobacteriaceae, Urinary Tract Infections, CLED, Port Sudan

\section{Introduction}

Definition: Any infection acquired in the community, that is, contrasted with those acquired in a health care facility (CROSS INFECTION). An infection would be classified as community-acquired if the patient had not recently been in a health care facility or been in contact with someone who had been recently in a health care facile.

Urinary tract infections (UTIs) are one of the most common infectious diseases, and nearly $10 \%$ of people will experience a UTI during their life time. The infections may be symptomatic or asymptomatic, and types of infection can result in serious sequelae if left untreated $4 \mathrm{M}$ [1].

Although several different micro organisms can cause
UTIs, including fungi and viruses, bacteria are the major causative organisms and are responsible for more than $95 \%$ of UTI cases [1].

The increase in the number of resistant and multiresistant (resistant to two and more antimicrobials) strains of bacteria is a major concern of health officials worldwide. Recently, bacterial resistance arising through the production of extended - spectrum beta - lactamases (ESBLs) has been recognized as a worldwide therapeutic problem [2]. The mechanisms of this resistance are often complex, and include production of $\beta$ - lactamases unregulated efflux pumps, and target site mutations [3].

In Gram-negative pathogens, $\beta$ - lactamases production remains the most important contributing factor to $\beta$-lactam 
resistance.1 DM Livermore, Bacterial resistance: origins, epidemiology, and impact, Clin Infect Dis 36 (suppl 1) (2003), pp. S11-S23. Full Text via CrossRef | View Record in Scopus | Cited By in Scopus (147) lactamases are bacterial enzymes that inactivate $\beta$-lactam antibiotics by hydrolysis, which results in ineffective compounds. One group of $\beta$ lactamases; extended - spectrum $\beta$ lactamases (ESBLs); have the ability to hydrolyse and cause resistance to various types of the newer $\beta$-lactam antibiotics, including the expandedspectrum (or third-generation) cephalosporins (e.g., cefotaxime, ceftriaxone and ceftazidime) and monobactams (e.g., aztreonam), but not the cephamycins (e.g., cefoxitin and cefotetan) and carbapenems (e.g., imipenem, meropenem, and ertapenem). Organisms that produce ESBL remain an important reason for therapy failure with cephalosporins and have serious consequences for infection control. That clinical microbiology laboratories detect and report ESBL - producing organisms is therefore important. Most ESBLs can be divided into three groups: TEM, SHV, and CTX-M types [4].

Wild type strains of are susceptible to all penicillins and cephalosporins. However, since 1990, a progressive increase of $\beta$-lactam. resistance, mediated by the production of acquired $\beta$-lactamases, has occurred in this species. [3] A. Endimiani, F. Luzzaro, G. Brigante, M. Perilli, G. Lombardi and G. Amicosante et al., Proteus mirabilis bloodstream infections: risk factors and treatment outcome related to the expression of extended-spectrum $\beta$-Lactamases, Antimicrob Agents Chemother 49 (2005), pp. 2598-2605. View Record in Scopus | Cited By in Scopus Various ESBLs, such as TEM, CTX-M, PER, and VEB - type ESBLs, have been described in P. mirabilis in separate geographic areas [5]. Available therapeutic options for the treatment of these antimicrobial -resistant organisms are severely limited because these organisms frequently display a multidrug resistant phenotype [6].

Treatment of UTI cases is often started empirically. Therapy is based on information determined from the antimicrobial resistance pattern of the urinary pathogens. However, because of the evolving and continuing antibiotic resistance phenomenon, regular monitoring of resistance patterns is necessary to improve guidelines for empirical antibiotic therapy.

\section{Materials and Method}

\subsection{Study Approach}

Study approach is to identify and isolate the possible causative organisms and the characterization of isolated strains using bio typing and anti bio gram.

\subsubsection{Study Type and Design}

Cross-sectional descriptive study.

\subsubsection{Study Area}

This work was carried out in Red Sea state. Urine collection and patients data were done in Port Sudan
Teaching Hospital (PTH), Sea Port Corporation Hospital, Prince Osman Digina Hospital. The laboratory investigation was done in the Port Sudan Alahlia college.

\subsubsection{Target Population}

Patients suffering from UTI signs and symptoms that attended the above mentioned hospitals were included in this study. The patients age varied from 2 years to 75 years.

\subsubsection{Data Collection}

Data were collected using structural interviewing questionnaire.

\subsection{Methodology}

\subsubsection{Sample Size and Collection}

According to standard method all specimen were examine to detect, and identify pathogens or their products using: microscopic examination of specimen to detect their motility, morphology and staining reaction. Also culture techniques to isolate pathogens in pure form and to identify them then test their antibiotic sensitivity and biochemical.

\subsubsection{Sampling}

Samples for community acquired UTI to investigate and diagnosis of microbial diseases (urine specimen).

Urine specimens were collected in case of urinary tract infection as following. A mid stream urine is obtained in a sterile container after cleaning the external genitalia with tap water and drying. Samples should reach the laboratory within one hour after voiding or kept refrigerated at $4 c^{\circ}$ to avoid multiplication of bacteria in urine then we do appropriate test to identify and isolate the causative organisms.

\subsection{Cultivation of Specimens}

Urine specimens were inoculated onto CLED medium by using sterile loop, then incubated aerobically at $37 \mathrm{c}^{\circ}$ over night.

\subsection{Examination of Bacterial Growth}

The primary culture on CLED medium that showed significant growth was examined for fermentation. The morphological character, size, shape, colour were observed and recorded.

\subsection{Interpretation of Culture Growth}

The culture growth obtained was interpreted as significant $\left(>10^{5} \mathrm{CFU} / \mathrm{ml}\right)$. Cultured of less than $(103 \mathrm{CFU} / \mathrm{ml})$ of urine was considered insignificant, while culture with no growth were considered negative. Significant cultures were further investigated.

\subsection{Purification of Bacterial Growth}

The isolates were streaked onto Nutrient agar and incubated overnight at $37^{\circ} \mathrm{C}$. The resultant growth was checked for purity and stored in Bijou bottle for further investigation. 


\subsection{Identification of the Isolated Bacteria}

\subsubsection{Colonial Morphology}

Colonial characteristic were observed on CLED medium after overnight incubation isolated organisms were growth on CLED medium.

\subsubsection{Biochemical Tests (Conventional Test)}

These are called biochemical tests because they are tests which identify the bacteria on the basis of the presence of certain enzymes and other biochemical properties.

\section{a. Oxidase Test}

In this test organism that produce the enzyme Cytochrome Oxidase oxidize phenylenediamine to a deep purple color. Oxidase reagent disc were placed on sterile Petri plates and rubbed with colony of the test organism using a piece of stick, and looked for a red-purple color with in $20 \mathrm{~min}$.

\section{b. Kliglar Iron Agar}

this medium was originally designed as a multi-test medium. It provides a low degree of sensitivity for $\mathrm{H} 2 \mathrm{~S}$ production (often required when differentiating members of the (Enterobacteriaceae). The medium is now used principally as a standard test for H2S. One disadvantage of multitest media is that chemical interaction-in this case acid production from fermentable sucrose-may inhibit blackening of the iron indicator. Some Citrobacter and Proteus species have this ability. KIA agar should be used in conjunction with a urease test to eliminate Proteus spp when screening for Salmonellae.

\section{c. Citrate Utilization Test}

This is for the ability of on organism to utilize citrate as the sole carbon and energy source for growth and an ammonium salt as sole source of nitrogen.

\section{d. Indole Test}

This test demonstrates the ability of certain bacteria to decompose the amino acid tryptophan to indole, which accumulates in the medium. Indole is then tested for by a colorimetric reaction with p- dimethylaminobenzaldehyde. Add $0.5 \mathrm{ml}$ kovacs reagent to see result.

\section{e. Urease Test}

Bacteria, particularly those growing naturally in an environment exposed to urine, may decompose urea by means of the enzyme urease The occurrence of this enzyme can be tested for by growing the organism in the presence of urea and testing for alkali production by means of a suitable $\mathrm{PH}$ indicator. An alternative method is to test for the production of ammonia from urea by means Nesslers reagent.

\section{f. Motility Test}

In semi solid agar media, motile bacteria (Swarm) and give a diffuse spreading growth that is easily recognized by the naked eye. Motility may thus be detected more easily than by the microscopical method.

\subsection{Antimicrobial Sensitivity Test}

All isolated microorganisms were subjected to antimicrobial sensitivity test using Modified Kirby-Bauer disc diffusion method.

\section{Results}

One hundred and fifty urine specimens were collected from patients suffering from UTI in the period from January 2017 to june 2017. The patients constitute 60 (40\%) males and $90(60 \%)$ females (Table 1$)$. The age of the patients ranged from two years to 75 years. Out of 150 patients $(0 \%)$ were infants, $8(5.3 \%)$ were children, $68(45.3 \%)$ were young adult, $42(28 \%)$ were adults and $32(21.3 \%)$ were elderly (Table 2). 120 (54\%) of specimens showed bacterial growth when cultured on Blood, MacConkey agar and CLED. Among these $32(21.3 \%)$ were lactose fermenters (LF) and the rest $17(11.3 \%)$ were non-lactose fermenters (Table 3).

Table 1. Distribution of specimens according to patients gender.

\begin{tabular}{lll}
\hline Gender & No of specimen & \% \\
\hline Male & 60 & 40 \\
Female & 90 & 60 \\
Total & 150 & 100 \\
\hline
\end{tabular}

Table 2. Distribution of specimens according to patients age group.

\begin{tabular}{llll}
\hline $\begin{array}{l}\text { Total Number of } \\
\text { specimens }\end{array}$ & Significant growth (\%) & $\begin{array}{l}\text { Insignificant } \\
\text { growth(\%) }\end{array}$ \\
\hline & $49(32.6 \%)$ & & \\
150 specimens & NLF(\%) & LF(\%) & $101(67.3 \%)$ \\
& $8 \% 17(11.3 \%)$ & $32(21.3 \%)$ & \\
\hline
\end{tabular}

Table 3. Significant and insignificant growth on MacConkey`s agar.

\begin{tabular}{ll}
\hline Age group & No. of specimens \\
\hline $0-5$ (Infant) & 0 \\
$5-12$ (Children) & $8(5.3 \%)$ \\
$16-30$ ( Young adult ) & $68(45.3 \%)$ \\
Elder (more than 46) & $32(21.3 \%)$ \\
\hline
\end{tabular}

Table 4. Activity of antibiotic on Enterobacteriaceae.

\begin{tabular}{|c|c|c|c|c|c|c|c|c|}
\hline Activity of antibiotic & IPM & CIP & $\mathbf{C}$ & $\mathbf{A K}$ & PI & TE & TI & CAZ \\
\hline Sensitive & $44(89)$ & $29(59)$ & $20(40)$. & $18(36)$. & 8 (16.) & $5(10)$. & $4(8.1)$ & $5(10)$. \\
\hline Resistance & $16(6)$. & $24(3)$ & $24(48)$ & $30(61)$ & $39(79)$ & $41(83)$ & 45 (91.) & $44(89.7)$ \\
\hline
\end{tabular}

Key:

(IPM) Imipenem, (CIP) ciprofloxacin, (C) chloramphenicol, (AK) amikacim, (PI) piperacllin (TE) tetracucline, (CAZ) Ceftazitim (Ti) Ticarcillin. 


\section{Discussion and Conclusion}

\subsection{Discussion}

This study was conducted to determine antimicrobials resistance profile of Enterobacteriaceae isolated. In this study out of one hundred and fifty urine specimens investigated, 49(32.6\%) showed significant bacterial growth, while 101(67.3\%) showed no growth and this result nearly similar with result obtained by Dr. Ummey Shahnaz Parvin [7], and disagree with the result was obtained by Muktikesh Dash et al, [8].

The present study females were more susceptible to infection 90 (60\%) while male less susceptible 60 (40\%) this result was agree to result obtained by Samiah HS Al-Mijalli et al [9].

The current study show that the, $68(45.3 \%)$ were young adult were most effect this result disagree with the result was obtained by Mohammed Akram, et., al [5]. Among these Escherichia coli 25 (51\%), Klebsiella spp 31(26\%), proteus spp 8(16\%), Citrobacter sp2(4\%), Salmonella spp 1(2.5\%). This result agree with result was obtained by Muktikesh Dash et al, [8],

In vitro activities of antimicrobial agents showed that the resistance rate of Enterobacteriaceae to ticarcillin (91.2\%) This result is disagrees with the result obtained by Nijssen et al, [10].

In present study the imipenem show highly sensitivity for UTIs and this result nearly similar with the result was obtained by Samiah HS Al-Mijall et al,. [9], and ciprofloxacin was show as $2^{\text {nd }}$ effective antimicrobial against urinary tract infection caused by Enterobacteriaceae this result agree with the result was obtained by J Hosp Infect. [11].

\subsection{Conclusion}

The study concluded that:

i. UTIs in a young adults, children and elder were more than infant.

ii. Most effective antibiotics for Enterobacteriaceae was imipenem and ciprofloxacin in due to low resistance.

iii. Chloramphenicol, amikacim and piperacllin are the second choice of antimicrobial therapy with resistance rate.

iv. Tetracycline, ceftazitim and Ticarcillin is less effective antimicrobial agents due to high resistance rate.

\section{Recommendations}

i. All urine specimens seen on routine work should investigated for culture and susceptibility tests.

ii. Introduction of the API 20 E for Enterobacteriaceae isolation is necessary in all Microbiology Laboratories.

iii. Establishment of antimicrobial policies and treatment guidelines are of major importance.

\section{References}

[1] Farajnia S, Mohammad Y, Reza G, Behrooz N and Ailar N, (2008). Causative agents and Antimicrobial Susceptibilities of Urinary Tract Infections in the Northwest of Iran. International Journal of Infectious Disease. Volume13. Issue2, pp 140-144.

[2] Bush K, (2008). Extended-spectrum $\beta$-lactamases in North America. Clin Microbiol Infect 14, 134-143.

[3] Sakoulas G and Moellering R, (2008). Increasing Antibiotic Resistance among Methicillin-resistant Staphylococcus aureus strains. Clin Infect Dis. 46: S360-S367.

[4] Johann D and Kevin B (2008). Extended-spectrum $\beta$ lactamase-producing Enterobacteriaceae:an emerging publichealth concern. The Lancet Infectious Disease, Volume 8, Issue 3, pp 159-166.

[5] Mahrouki Achour N, Chouchan C, Moussa M, and Belhadj O, (2008). Identification of Plasmid-encoded Extended Spectrum $\beta$-lactamases Produced by a clinical Strain of Proteus mirabilis. Pathologie Biologie. Volume 57, Issue3, pp 55-59.

[6] George G, Mel D, Kim N, Aleksandra W, Patricia J, James A, Philippe L, Andrew W, Michael R, and Daryi J, (2008). Antimicrobial Susceptibility of 3931 Organisms Isolated from Intensive Care Units in canada. Diagnostic Microbiology and Infectious Disease. Volume 62, Pages 67-80.

[7] Mims Cedric, dockrell. Hazel. M, Goering. V, Roitt. Ivan, Wakelin. Derek and Zuckerman. Mark. (2004). Medical microbiology, third edition, ELSEVIER MOSBY.

[8] Tsai L, Clifford M, Yih R, Pei C, Hui Y, Jui F, Monto H and TSAR Participating Hospitals, (2003). The Status of Antimicrobial Resistance in Taiwan among Gram-negative Pathogens: the Taiwan Surveillance of Antimicrobial Resistance (TSAR) Program. Diagnostic Microbiology and Infectious Disease. Volume 48, Issue 3, pp 211-219.

[9] Sita N, Merode T, Bartelds A, and Stobberingh E, (2005), Antibiotic Treatment and Resistance of Unselected Uropathogens in the Elderly. International Journal of Antimicrobial Agent. Volume27. Issue3, pp 236-241.

[10] Nijssen, Florijn A, Bonten M, Schmitz F, Verhoef J and Fluit A, (2004). Beta-lactam Susceptibilities and Prevalence of ESBL-producing Isolates among more than 5000 European Enterobacteriaceae isolates, University Medical Center Utrecht. International Journal of Antimicrobial Agent. Volume24. Issue6, pp 585-591.

[11] Farmer J, 1999. Enterobacteriaceae, pp.442-458. In P. R. Murray, E. J. Baron, M. A. Pfaller, F. C. Tenover, and R. H. Yolken (ed.), Manual of clinical microbiology, 7th ed. ASM Press, Washington, D. C. 